\title{
An Atoh1 CRE Knock-In Mouse Labels Motor Neurons Involved in Fine Motor Control
}

\author{
Osita W. Ogujiofor, ${ }^{1, *}$ Iliodora V. Pop, ${ }^{1, *}$ Felipe Espinosa, ${ }^{1}$ Razaq O. Durodoye, ${ }^{1}$ \\ Michael L. Viacheslavov, ${ }^{1}$ Rachel Jarvis, ${ }^{1}$ - Mark A. Landy, ${ }^{1}$ Channabasavaiah B. Gurumurthy, ${ }^{2,3}$ and \\ Helen C. Lai ${ }^{1}$
}

\section{https://doi.org/10.1523/ENEURO.0221-20.2021}

${ }^{1}$ Department of Neuroscience, University of Texas Southwestern Medical Center, Dallas, TX 75390, ${ }^{2}$ Mouse Genome Engineering Core Facility, University of Nebraska Medical Center, Omaha, NE 68198, and ${ }^{3}$ Department of Pharmacology and Experimental Neuroscience, College of Medicine, University of Nebraska Medical Center, Omaha, NE 68198

\begin{abstract}
Motor neurons (MNs) innervating the digit muscles of the intrinsic hand (IH) and intrinsic foot (IF) control fine motor movements. The ability to reproducibly label specifically IH and IF MNs in mice would be a beneficial tool for studies focused on fine motor control. To this end, we find that a CRE knock-in mouse line of Atoh1, a developmentally expressed basic helix-loop-helix (bHLH) transcription factor, reliably expresses CRE-dependent reporter genes in $\sim 60 \%$ of the IH and IF MNs. We determine that CRE-dependent expression in IH and IF MNs is ectopic because an Atoh1 mouse line driving FLPo recombinase does not label these MNs although other Atoh1-lineage neurons in the intermediate spinal cord are reliably identified. Furthermore, the CRE-dependent reporter expression is enriched in the IH and IF MN pools with much sparser labeling of other limb-innervating MN pools such as the tibialis anterior (TA), gastrocnemius (GS), quadricep (Q), and adductor (Ad). Lastly, we find that ectopic reporter expression begins postnatally and labels a mixture of $\alpha$ and $\gamma$-MNs. Altogether, the Atoh1 CRE knock-in mouse strain might be a useful tool to explore the function and connectivity of MNs involved in fine motor control when combined with other genetic or viral strategies that can restrict labeling specifically to the IH and IF MNs. Accordingly, we provide an example of sparse labeling of IH and IF MNs using an intersectional genetic approach.
\end{abstract}

Key words: Atoh1; fine motor control; motor neurons; spinal cord

\section{Significance Statement}

Motor neurons (MNs) of the intrinsic hand (IH) and intrinsic foot (IF) are reproducibly labeled in an ectopic manner postnatally using a CRE knock-in mouse line of the basic helix-loop-helix (bHLH) transcription factor Atoh1, serving as a useful genetic tool for future studies of fine motor control.

\section{Introduction}

Motor neurons (MNs) innervating the muscles in the digits of the hand allow for exquisite control of fine motor movements required for dexterous skills, such as writing or sewing. Primates are known for their ability to precisely control individual digits of the hand, but skillful movements are not limited to the hand, as people born without

\footnotetext{
Received May 25, 2020; accepted January 8, 2021; First published January 19, 2021.

The authors declare no competing financial interests.
}

arms are able to dexterously manipulate tools with their toes (Shubin et al., 1997; Sustaita et al., 2013; DempseyJones et al., 2019). Precisely how MNs innervating the intrinsic hand $(\mathrm{IH})$ and intrinsic foot (IF) muscles may differ in terms of function and connectivity compared with MNs that innervate limb muscles is unknown. Presumably, $\mathrm{IH}$ and IF MNs have unique properties and connectivity that

Author contributions: H.C.L. designed research; O.W.O., I.V.P., F.E., R.O.D., M.L.V., R.J., and M.A.L. performed research; C.B.G. contributed unpublished reagents/analytic tools; O.W.O., I.V.P., and H.C.L. analyzed data; O.W.O., I.V.P., F.E., and H.C.L. wrote the paper. 
contribute to their role in fine motor control, however, examination of the function of specifically $\mathrm{IH}$ and IF MNs is underexplored.

In part, lack of exploration of the IH and IF MNs is because of an inability to genetically distinguish these MNs from other limb MNs in mice. Mice also demonstrate remarkable manipulative dexterity when performing fine motor tasks such as eating dried pasta or grasping a pellet (Tennant et al., 2010; Yoshida and Isa, 2018) and, thus, represent a model organism to study fine motor skills. Developing a genetic tool that labels IH and IF MNs would have significant utility in interrogating the function and circuitry of fine motor control. To this end, we found that digit-innervating $\mathrm{MNs}$ were labeled using $\mathrm{CRE}-\mathrm{loxP}$ recombination driven by the bHLH transcription factor atonal homolog 1, Atoh1, a transiently expressed gene in the dorsal-most part of the developing neural tube that is also known to specify excitatory $\left(\right.$ Vglut $^{+}$) spinal cord neurons that project rostrally to the hindbrain (Bermingham et al., 2001; Gowan et al., 2001; Sakai et al., 2012; Yuengert et al., 2015; Pop et al., 2020).

Here, we explore the features of the digit-innervating MNs labeled by Atoh1 CRE-LoxP recombination. We find that labeling of $\mathrm{IH}$ and IF MNs is ectopic because an Atoh1 mouse line expressing FLPo recombinase that we developed in the lab does not label IH and IF MNs, although it does label the expected Atoh1-lineage neurons in the intermediate spinal cord. The Atoh1 CRE knock-in mouse labels a mixture of $\alpha$-MNs and $\gamma$-MNs in the IH and IF MN pools postnatally, while sparsely labeling other limb-innervating MNs. We further endeavor to show the utility of the Atoh1 CRE knock-in mouse using an intersectional genetic approach and discuss possible future methodologies to interrogate fine motor function and circuitry using this mouse strain.

\section{Materials and Methods}

\section{Mouse strains}

The following mouse strains were used: Atoh $1^{\mathrm{Cre} /+}$ knock-in (Yang et al., 2010), R26 ${ }^{\text {LSL-tdTom/+ }}$ (Ai14; Madisen

This work was supported by NIH Grants R35HG010719 and R21GM129559 (to C.B.G.) and R21 NS099808 and R01 NS100741 (to H.C.L.).

${ }^{*}$ O.W.O. and I.V.P. contributed equally to this work.

Acknowledgements: We thank Sam Pfaff for the HB9 antibody; Tom Jessell for the Cpne4 and Fign antibodies; Lin Gan for the Atoh $1^{\text {Cre/+ }}$ knock-in mouse; Gord Fishell and Rob Machold for sharing the Chat ${ }^{I R E S-F L P O}$ mouse; Mark Behlke and Sarah Jacobi from Integrated DNA Technologies for providing preproduction megamers; Thomas Südhof for the Syntaxin1 antibody; the Neuroscience Microscopy Facility which is supported by the University of Texas Southwestern (UTSW) Neuroscience Department and the UTSW Peter O’Donnell, Jr. Brain Institute, Katherine Casey, Clayton Trevino, Megan Goyal, and Adelaide Brooks for technical assistance; and Peter Tsai, Weichun Lin, Jon Terman, Euiseok Kim, and Jane Johnson for helpful discussions and careful reading of this manuscript.

Correspondence should be addressed to Helen C. Lai at helen.lai@ utsouthwestern.edu.

https://doi.org/10.1523/ENEURO.0221-20.2021

Copyright @ 2021 Ogujiofor et al.

This is an open-access article distributed under the terms of the Creative Commons Attribution 4.0 International license, which permits unrestricted use, distribution and reproduction in any medium provided that the original work is properly attributed.

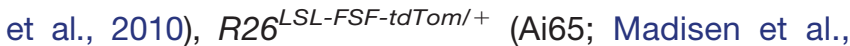
2015), $R 26^{\text {FSF-tdTom/+ }}$ was generated using CAG-Cre (Sakai and Miyazaki, 1997) crossed to Ai65, R26 FSF-EGFP/+ (named $R C E:: R F r t$ in Miyoshi et al., 2010), and Chat ${ }^{\text {RES-FLPo (Allaway }}$ et al., 2020). All mice were outbred and thus, are mixed strains (at least C57BI/6J and ICR). Atoh $1^{\mathrm{Cre} /+}$ knock-in mice crossed to reporter mice were screened for "dysregulated" expression as previously reported (Yuengert et al., 2015). All animal experiments were approved by the Institutional Animal Care and Use Committee at University of Texas Southwestern.

The Atoh1 $1^{\text {22A-FLPo/+ }}$ mouse was generated using the Easi-CRISPR approach (Quadros et al., 2017). Briefly, a long single stranded DNA cassette consisting of a viral peptide self-cleaving sequence [porcine teschovirus-1 2A (P2A); Kim et al., 2011] and the codon optimized flippase recombinase sequence (FLPo) were inserted after the last amino acid codon and before the stop codon of Atoh1. C57Bl/6N zygotes were microinjected with ribonucleoprotein complexes of Cas9 protein, tracrRNA, and crRNA (5'-TGACTCTGATGAGGCCAGTT-3') along with a ssDNA megamer for homologous recombination (1497 bp containing 60 bp each 5' and 3' homology arms and the P2AFLPo sequence; reagents were procured from IDT, microinjection service was provided by the UTSW Transgenic Mouse Core Facility). Assembling CRISPR reagents and microinjections were performed as previously described (Jacobi et al., 2017; Miura et al., 2018). The live born mice were first screened for insertion of the P2A-FLPo sequence and of those that were positive, one of the mice contained the full-length cassette. The cassette contained a minor mutation at the end of FLPo (the last isoleucine amino acid was changed to a serine), which could have occurred possibly because of an imprecise DNA repair event. Nevertheless, this amino acid change does not seem to affect the enzymatic function of FLPo. For genotyping, wild-type (WT) 321-bp and mutant 642-bp PCR products were detected using the following primers: WT forward 5'-CCCTAACAGCGATGATGGCACAGAAGG-3', WT reverse 5' -GGGGATTGGAAGAGCTGCAGCCGTC-3', and MUT reverse 5'-CGAACTGCAGCTGCAGGCTGGA CACG-3'. Note that because the P2A sequence selfcleaves near its $C$ terminus, 21 amino acids of the P2A sequence are fused to the $\mathrm{C}$ terminus of $\mathrm{ATOH} 1$.

\section{Tissue processing}

Embryos were timed as embryonic day (E) 0.5 on the day the vaginal plug was detected and $\mathrm{PO}$ on the day of birth. Pregnant females were euthanized with $\mathrm{CO}_{2}$ and cervical dislocation, embryos dissected out of the uterus, and spinal cords dissected out. Embryonic spinal cords (E14.5) were fixed in 4\% paraformaldehyde (PFA)/PBS for 2-3 h at $4^{\circ} \mathrm{C}$. Early postnatal animals (postnatal day 7 (P7) or younger) were cooled on ice, decapitated, their spinal cords dissected out, and fixed in $4 \%$ PFA/PBS for $2 \mathrm{~h}$ at $4^{\circ} \mathrm{C}$. Mice older than P14 were anesthetized with avertin (2,2,2-tribromoethanol; 0.025-0.030 $\mathrm{ml}$ of $0.04 \mathrm{M}$ avertin in 2-methyl-2butanol and distilled water/g mouse) and transcardially perfused, first with $0.012 \% \mathrm{w} / \mathrm{v}$ Heparin/PBS and then $4 \%$ PFA/PBS. A dorsal or ventral laminectomy exposed the 

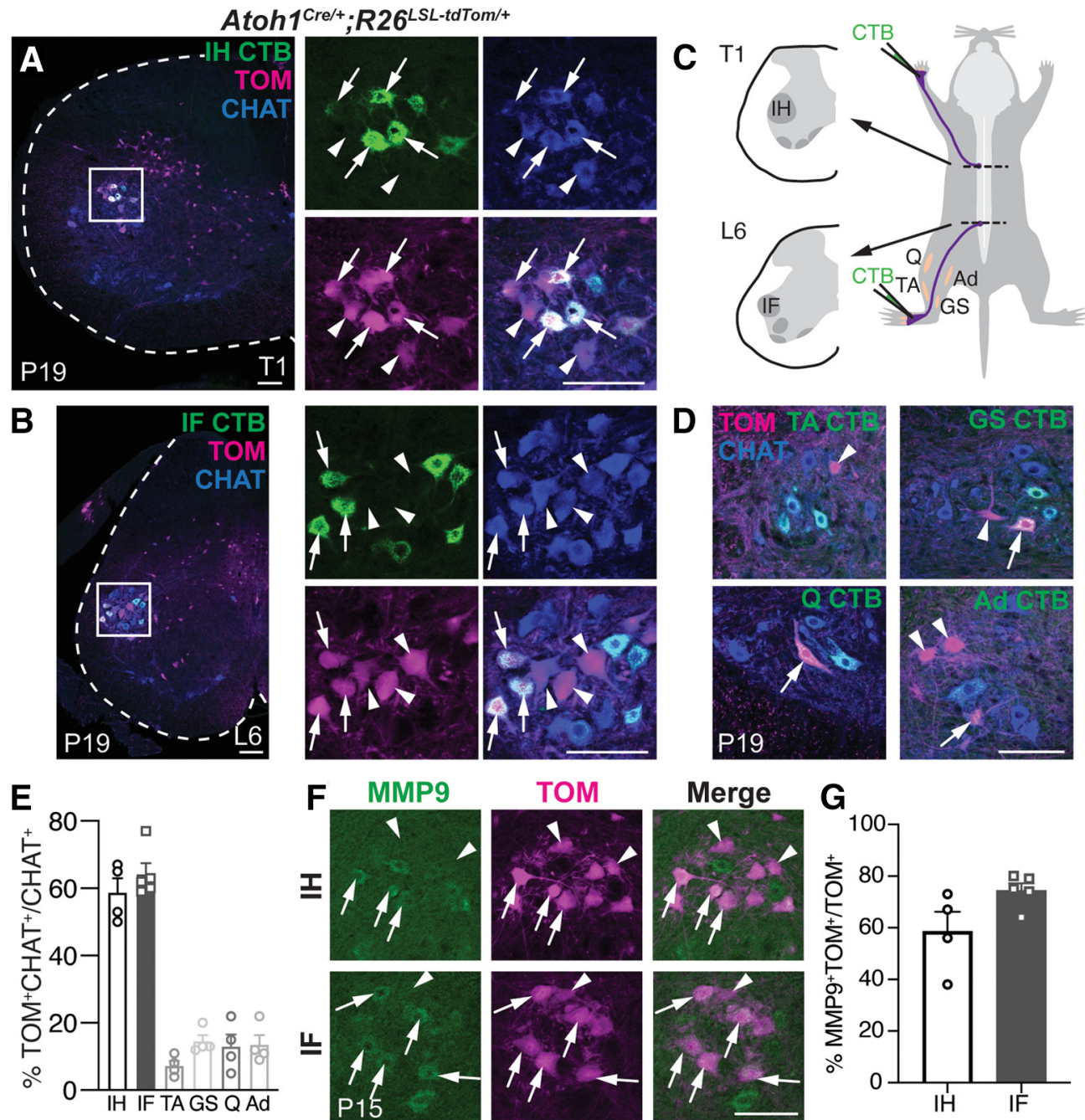

\section{Merge}
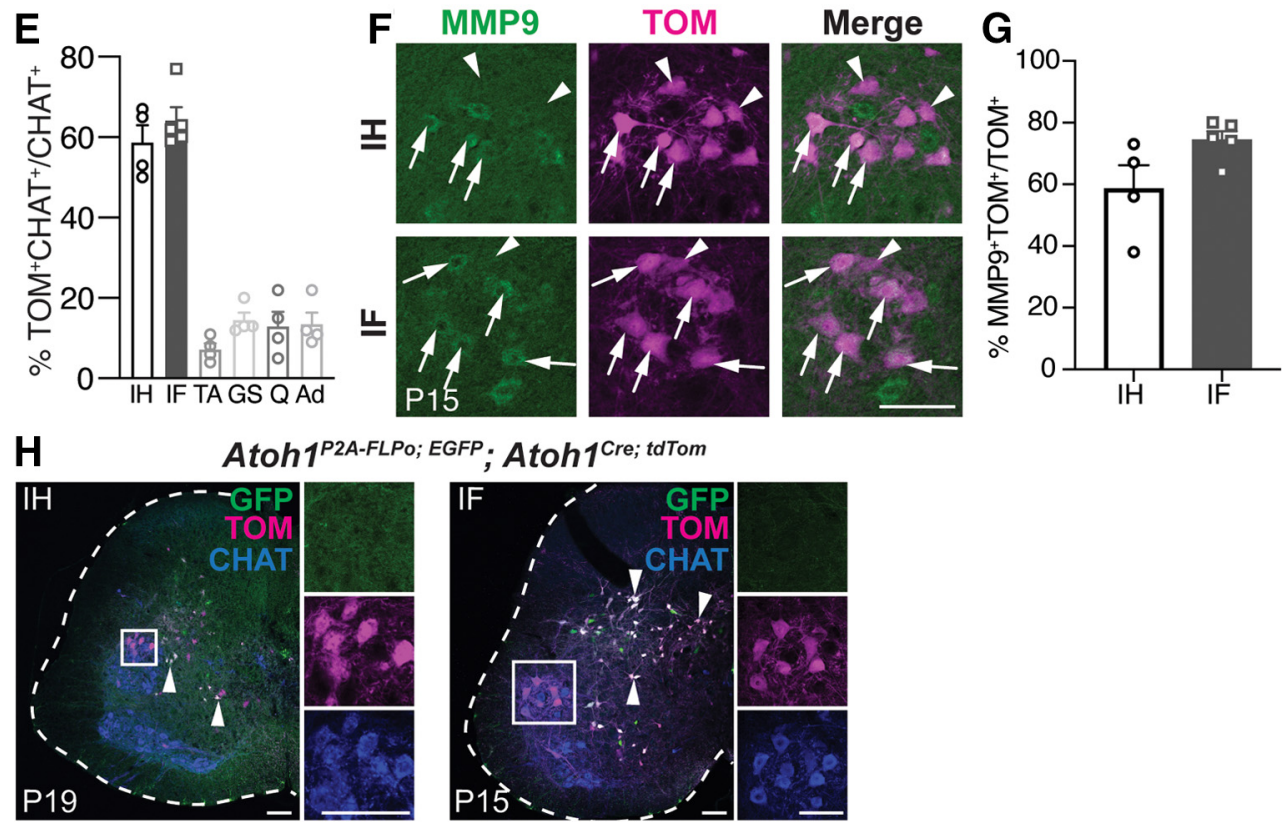

Figure 1. The Atoh $1^{\mathrm{Cre} /+}$ knock-in mouse line labels the IH and IF MN pools. $\boldsymbol{A}, \boldsymbol{B}$, Injection of the retrograde tracer $\mathrm{CTB}-488$ into the forepaw and hindpaw labels the IH and IF MN pools, which are labeled with tdTomato (TOM) in Atoh $1^{\mathrm{Cre} /+} \mathrm{knock}-\mathrm{in}$ mice. MN pools are identified with ChAT antibody. Arrows, $\mathrm{CTB}^{+} \mathrm{TOM}^{+} \mathrm{ChAT}^{+}$; arrowheads, $\mathrm{CTB}^{-} \mathrm{TOM}^{+} \mathrm{ChAT}^{+}$. C, Diagram of CTB-488 injections into the IH and IF MN pools, which are located at T1 and L6. D, Injection of CTB-488 into the TA, GS, Q, and Ad muscles retrogradely labels those MN pools, which are sparsely labeled with TOM in Atoh $1^{\mathrm{Cre} /+}$ knock-in mice. Arrows, $\mathrm{CTB}^{+} \mathrm{TOM}^{+} \mathrm{ChAT}^{+}$; arrowheads, $\mathrm{CTB}^{-} \mathrm{TOM}^{+} \mathrm{ChAT}^{+}$. E, Percentage of the IH, IF, TA, GS, Q, and Ad MN pools that are labeled $\mathrm{TOM}^{+}$in Atoh $^{\mathrm{Cre} /{ }^{+}}$ knock-in mice. $\boldsymbol{F}, \boldsymbol{G}$, Some of the $\mathrm{TOM}^{+} \mathrm{IH}$ MNs and IF MNs are fast twitch MNs $\left(\mathrm{MMPg}^{+}\right)$. Arrows, $\mathrm{MMPg}^{+} \mathrm{TOM}^{+}$; arrowheads, $\mathrm{MMP9}^{-\mathrm{TOM}^{+}} . \boldsymbol{H}$, Atoh1-lineage neurons in the intermediate spinal cord have extensive overlap in Atoh $1^{\text {P2A-FLPo; EGFP }}$ mice crossed

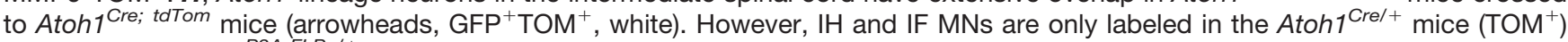
and not in the Atoh $1^{P 2 A-F L P O /+}$ mice (GFP; inset). P, postnatal; T, thoracic; L, lumbar. Scale bars: $100 \mu \mathrm{m}$.

spinal cord to the fixative. The spinal cords were fixed for 2 $\mathrm{h}$ and the brains overnight at $4^{\circ} \mathrm{C}$. Tissue was washed in PBS for at least $1 \mathrm{~d}$ and cryoprotected in $30 \%$ sucrose dissolved in deionized water. Tissue was marked with $1 \%$
Alcian Blue in 3\% acetic acid on one side to keep orientation and were then embedded in OCT (Tissue-Tek Optimal Cutting Temperature compound). Tissue was sectioned using a Leica CM1950 Cryostat. 

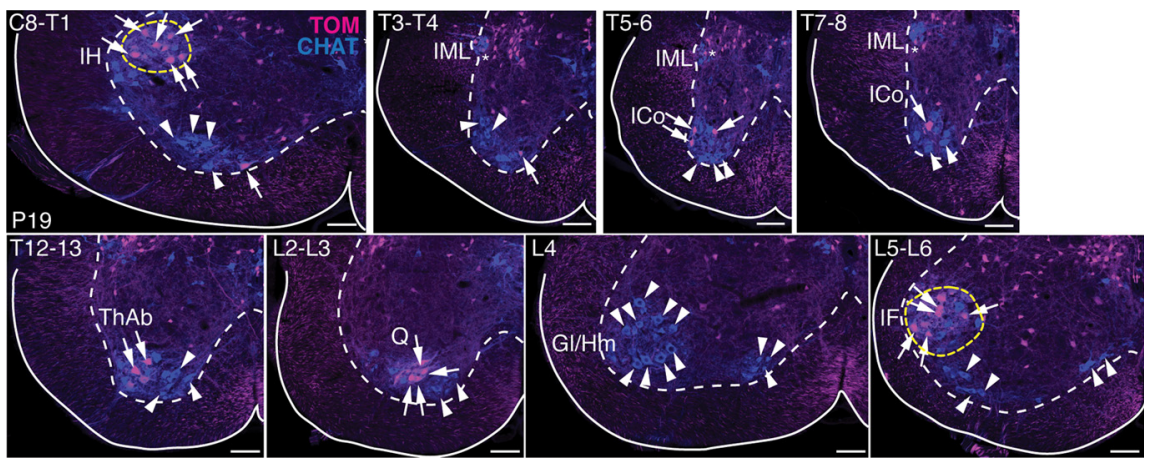

Figure 2. Distribution of MNs labeled in the Atoh $1^{\mathrm{Cre} /+}$ knock-in mouse throughout the spinal cord. Representative images throughout the rostral-caudal axis of Atoh $1^{\mathrm{Cre} /+}$ knock-in mice crossed to the TOM reporter mouse show that TOM labels MNs mainly in the IH and IF MN pools (yellow dashed lines) with sparser labeling of MNs in other MN pools (arrows). Some MN pools have no $\mathrm{TOM}^{+}$expression (arrowheads). $\mathrm{TOM}^{+} \mathrm{ChAT}^{+}$cells in the IML are sympathetic pre-MNs (asterisks). IML, intermediolateral nucleus; ICo, intercostal; ThAb, thoracic abductor; Q, quadricep; Gl, gluteus; Hm, Hamstring. Scale bars: $100 \mu \mathrm{m}$.

\section{Immunohistochemistry and confocal imaging}

Cryosections $(30 \mu \mathrm{m})$ were blocked with PBS/1-3\% normal goat or donkey serum/0.3\% Triton X-100 (Sigma) for up to $1 \mathrm{~h}$ at room temperature (RT) and incubated overnight with primary antibody at $4^{\circ} \mathrm{C}$. After washing three times with PBS, the appropriate secondary antibody (Alexa Fluor 488,567 , and/or 647, Invitrogen) was incubated for $1 \mathrm{~h}$ at RT. Sections were rinsed three times in PBS, mounted with Aquapolymount (Polysciences Inc.), and coverslipped (Fisher). The following primary antibodies and dilutions were used: 1:500 rabbit anti-dsRed (Clontech), 1:100 goat anti-choline acetyltransferase (ChAT; Millipore Sigma), 1:1000 rabbit anti-matrix metalloproteinase-9 (MMP9; Abcam), 1:8000 rabbit anti-HB9 (gift of Sam Pfaff, Salk Institute), 1:3000 guinea pig anti-copine-4 (CPNE4) and 1:8000 guinea pig anti-fidgetin (FIGN; gifts of Tom Jessell, Columbia University), 1:500 mouse anti-NEUN (Millipore Sigma), 1:100 mouse anti-ERR3 (R\&D Systems), 1:3000 $\alpha$-bungarotoxin (BTX) 488 (Invitrogen), 1:1000 rabbit anti-Syntaxin1 (gift of Thomas Südhof, Stanford University), and 1:1000 guinea pig anti-vesicular glutamate transporter 1 (VGLUT1; Millipore Sigma). Sections were referenced to the Christopher Reeves Spinal Cord Atlas (Watson et al., 2009).

Fluorescent images were taken on a Zeiss LSM710 or LSM880 confocal microscope with an appropriate optical slice $(0.5-10 \mu \mathrm{m})$ depending on the image. Images were pseudocolored using a magenta/green/blue or magenta/ yellow/cyan color scheme using Adobe Photoshop (Adobe) or Fiji (Schindelin et al., 2012).

\section{CTB muscle injections}

Mice aged P14 were anesthetized using isoflurane and prepared for injections into muscle. An approximate total of 500-750 nl of cholera toxin subunit B (CTB) Alexa Fluor 488 or 647 conjugate (Invitrogen; Nanoject II, Drummond Scientific) was injected into two to three different locations in the left forepaw (IH MN pool) or hindpaw (IF MN pool) in $50.6 \mathrm{nl}$ increments. For injections into the tibialis anterior (TA), gastrocnemius (GS), quadricep (Q), or adductor (Ad), the area of the skin above the muscle was shaved and $70 \%$ ethanol and betadine (Avrio Health L.P.) applied. An incision was made above the muscle and 500-750 $\mathrm{nl}$ of the CTB-conjugated Alexa Fluor was injected into three to four different locations directly into the muscle. The incision was closed with surgical glue (Henry Schein Medical). Carprofen $(5 \mathrm{mg} / \mathrm{kg})$ was administered daily $3 d$ after surgery. Spinal cords were harvested $5 d$ after injection. For injections at P0, mice were anesthetized with isoflurane and injected with $<250 \mathrm{nl}$ CTB-488 or 647 in one or two different locations of the forepaw and hindpaw and harvested $3 \mathrm{~d}$ later.

\section{Experimental design and statistical tests}

All details for number of sections counted, biological replicates, and male and female tissue analyzed are given in Results. No statistical tests were required as quantitation of the percentage of particular markers in any given $\mathrm{MN}$ pool were not directly compared with each other. Mean \pm SEM are reported throughout. For samples with $n=2$, the SEM is equal to half of the range between the two data points.

\section{Results}

\section{Atoh $1^{\text {Cre/ }}{ }^{+}$knock-in mice label MN pools involved in fine motor control}

We observed using CRE-lineage tracing strategies (Atoh $1^{\mathrm{Cre} /+}$ knock-in mice crossed to tdTomato (TOM) reporter mice (R26 LSL-Tom, Ai14)) (Yang et al., 2010; Madisen et al., 2010) that subsets of MNs expressing ChAT were labeled in the spinal cord (Fig. $1 A, B$, arrows and arrowheads). Based on the anatomic location of the MN pools along the rostral-caudal axis, we predicted that the Atoh ${ }^{\mathrm{Cre} /+}$ line labeled MNs of the IH and IF in thoracic 1 (T1) and lumbar 6 (L6) areas of the spinal cord (Fig. 1C; Watson et al., 2009). We injected the forepaw and hindpaw with the retrograde tracer CTB conjugated to Alexa Fluor 488 (CTB-488) and verified that the IH and IF MN pools were Atoh $1^{\mathrm{Cre} /+} \mathrm{TOM}^{+} \mathrm{MNs}$ (Fig. $1 A, B$, arrows). Note that the $\mathrm{TOM}^{+}$cell bodies in the intermediate spinal cord are from other Atoh1-lineage interneurons 


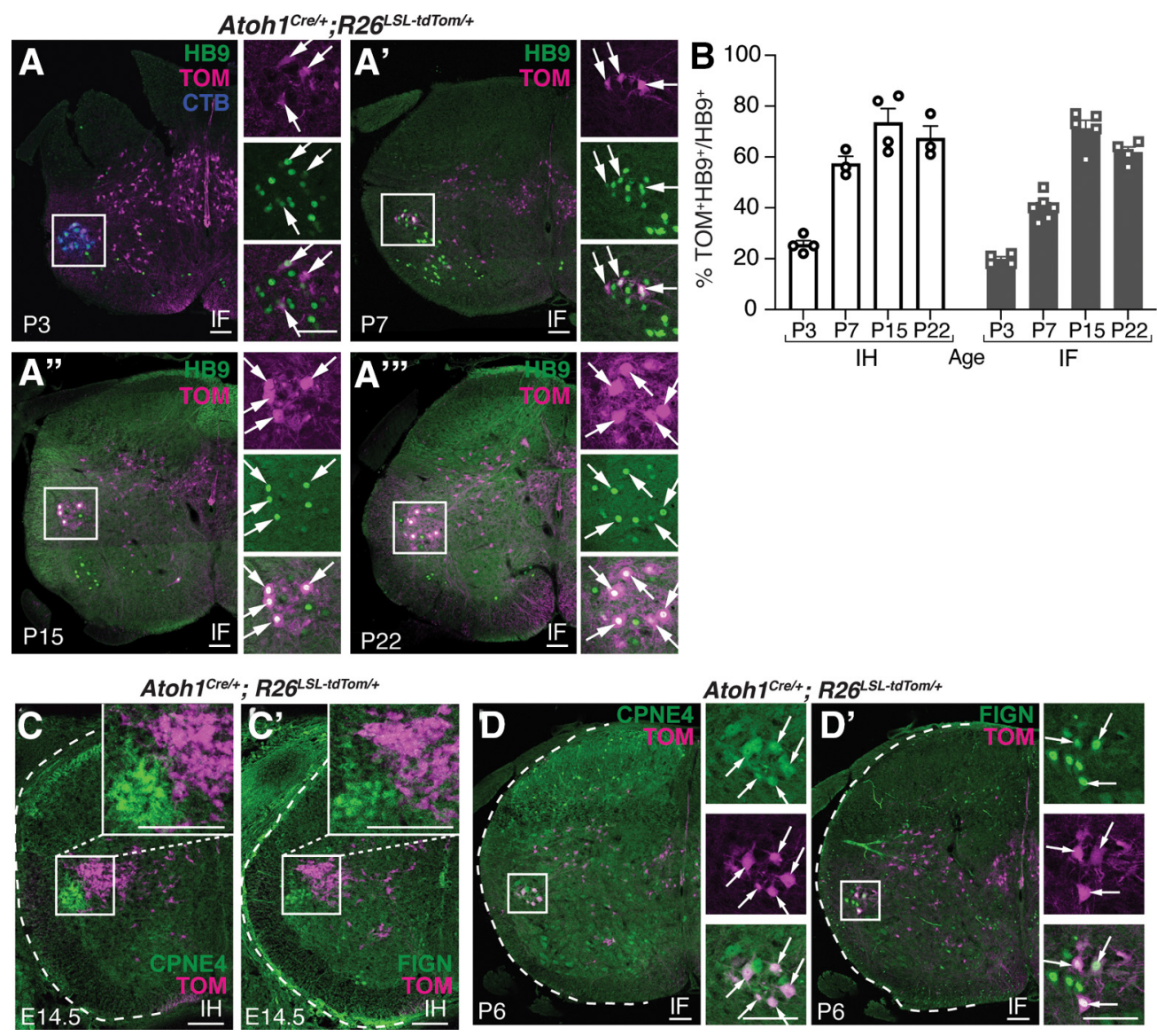

Figure 3. The Atoh $1^{\mathrm{Cre} /+}$ knock-in mouse labels IH and IF MN pools postnatally. $\boldsymbol{A}-\boldsymbol{A}^{\text {'”', }}$ TOM ${ }^{+}$labeling of the IF MN pool at several postnatal time points. $\mathrm{TOM}^{+} \mathrm{HB}^{+}$neurons, arrows. In $\boldsymbol{A}$, CTB (blue) was injected into the hindpaw to identify the IF MN pool. In $\boldsymbol{A}^{\prime}-$ $\boldsymbol{A}^{\prime \prime \prime}$, the IF MN pool was identified by location in the lumbar spinal cord. B, Percentage of $\mathrm{TOM}^{+}$neurons in the IH or IF MN pools at several postnatal time points. The IH and IF MN pools were identified by CTB injection into the forepaw and hindpaw at PO and tissue harvested $3 \mathrm{~d}$ later for the P3 time point. IH and IF MN pools for P7, P15, and P22 were identified by location in the lumbar spinal cord. C-C', At E14.5, $\mathrm{TOM}^{+}$neurons are CPNE4 ${ }^{-}$and $\mathrm{FIGN}^{-}$. D-D', At P6, $\mathrm{TOM}^{+}$neurons are CPNE4 ${ }^{+}$and FIGN ${ }^{+}$(arrows). Scale bars: $100 \mu \mathrm{m}$.

involved in the proprioceptive system (Yuengert et al., 2015; Pop et al., 2020).

To see whether the labeling of $A t o h 1^{\mathrm{Cre} /+} \mathrm{TOM}^{+} \mathrm{MNs}$ was specific to the IH and IF MN pools, we injected CTB488 into the TA, GS, Q, and Ad muscles and found that those MN pools had much fewer TOM ${ }^{+}$MNs (Fig. 1D, arrows and arrowheads). In addition, we sampled sections throughout the rostral-caudal axis of the spinal cord in Atoh $1^{\text {Cre/ }}$ mice and found that few other MN pools had $\mathrm{TOM}^{+}$expression (Fig. 2, arrows). Altogether, the $\mathrm{TOM}^{+}$ MNs labeled $\sim 60 \%$ of the IH and IF MN pools at $\mathrm{P} 19[\mathrm{IH}$ : $59 \pm 4 \%, n=4,1: 3$ male $(\mathrm{M})$ : female $(\mathrm{F})$, four to eight half sections $/ n$; IF: $64 \pm 3 \%, n=5,1: 4 \mathrm{M}: \mathrm{F}$, two to eight half sections $/ n$; TA: $7 \pm 2 \%, n=4,2: 2 \mathrm{M}: \mathrm{F}$, two half sections/ $n$; GS: $14 \pm 2 \%, n=4,2: 2 \mathrm{M}: \mathrm{F}$, two to four half sections $/ n$; $\mathrm{Q}: 13 \pm 4 \%, n=4,2: 2 \mathrm{M}: \mathrm{F}$, one to three half sections $/ n$; Ad: $13 \pm 3 \%, n=4,2: 2 \mathrm{M}: \mathrm{F}$, one to six half sections $/ n$. MN areas located by CTB-488 injection into appropriate muscle group; Fig. 1E. We estimate that the total number of $\mathrm{ChAT}^{+} \mathrm{MNs}$ in the IH and IF MN pools at P19 on one side is $410 \pm 72$ neurons for the $\mathrm{IH} \mathrm{MN}$ pool $(n=3,1: 2 \mathrm{M}: \mathrm{F}$, three to four half sections $/ n$ ) and $337 \pm 7$ SEM neurons for the IF MN pool ( $n=3,0: 3 \mathrm{M}: \mathrm{F}$, three half sections $/ n$ ). Counts of sections represented a tenth of the $\mathrm{MN}$ pool, so the estimated total number of $\mathrm{ChAT}^{+}$neurons were the final counts multiplied by ten. Because the $\mathrm{TOM}^{+} \mathrm{MNs}$ comprised $\sim 60 \%$ of the IH and IF MN pools, we hypothesized that the Atoh $1^{\mathrm{Cre} /+}$ mice might be labeling a particular functional class of MNs such as fast or slow twitch MNs. However, we found that only a subset of the $\mathrm{IH}$ and IF $\mathrm{TOM}^{+}$MNs were labeled by the marker for fast MNs, MMP9, indicating that the IH and IF TOM ${ }^{+} \mathrm{MNs}$ are a mixture of fast and slow MNs $(\mathrm{IH}: 59 \pm 8 \%, n=4,3: 1 \mathrm{M}: \mathrm{F}$, three half sections $/ n$; IF: $74 \pm 3 \%, n=5,3: 2 \mathrm{M}: F$, three half sections/n; Fig. 1F,G, arrows; Kaplan et al., 2014).

Developmentally, all cholinergic MNs derive from a progenitor domain expressing the basic helix-loop-helix (bHLH) oligodendrocyte transcription factor 2 (Olig2) in the ventral neural tube (Lu et al., 2002), not the transiently-expressed Atoh1 dorsal-most progenitor domain (Lai et al., 2016). Therefore, we tested whether Atoh1 was expressed in MNs by in situ hybridization (Gowan et al., 2001 for ISH probe) and RNAscope of Atoh1 at P14-P15 and P22, ages that we knew had $\mathrm{TOM}^{+}$expression, but 

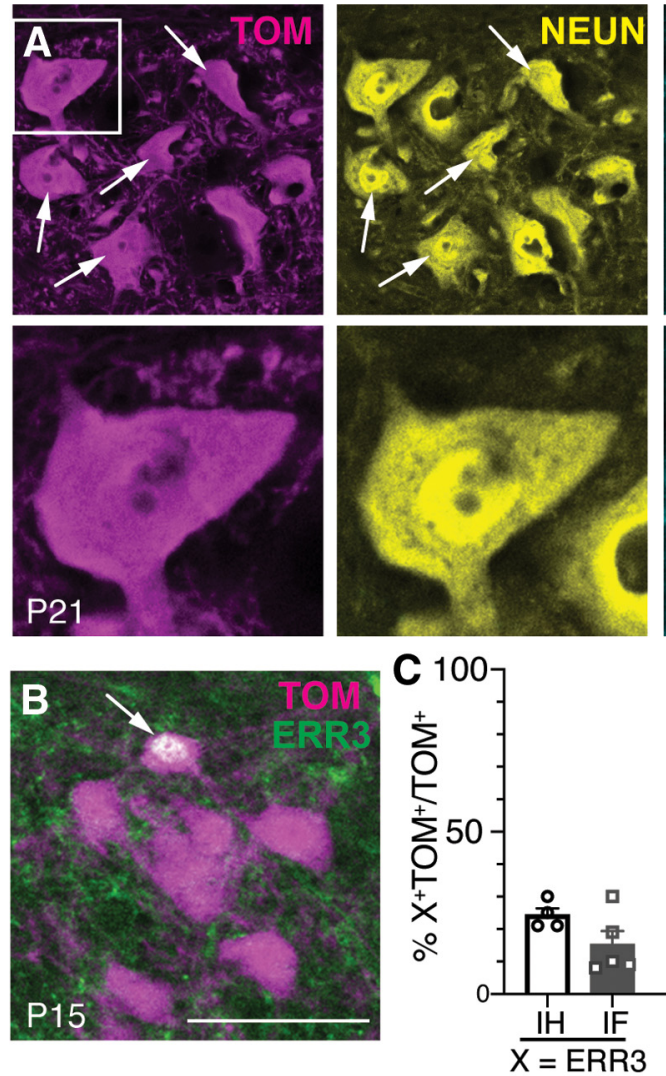
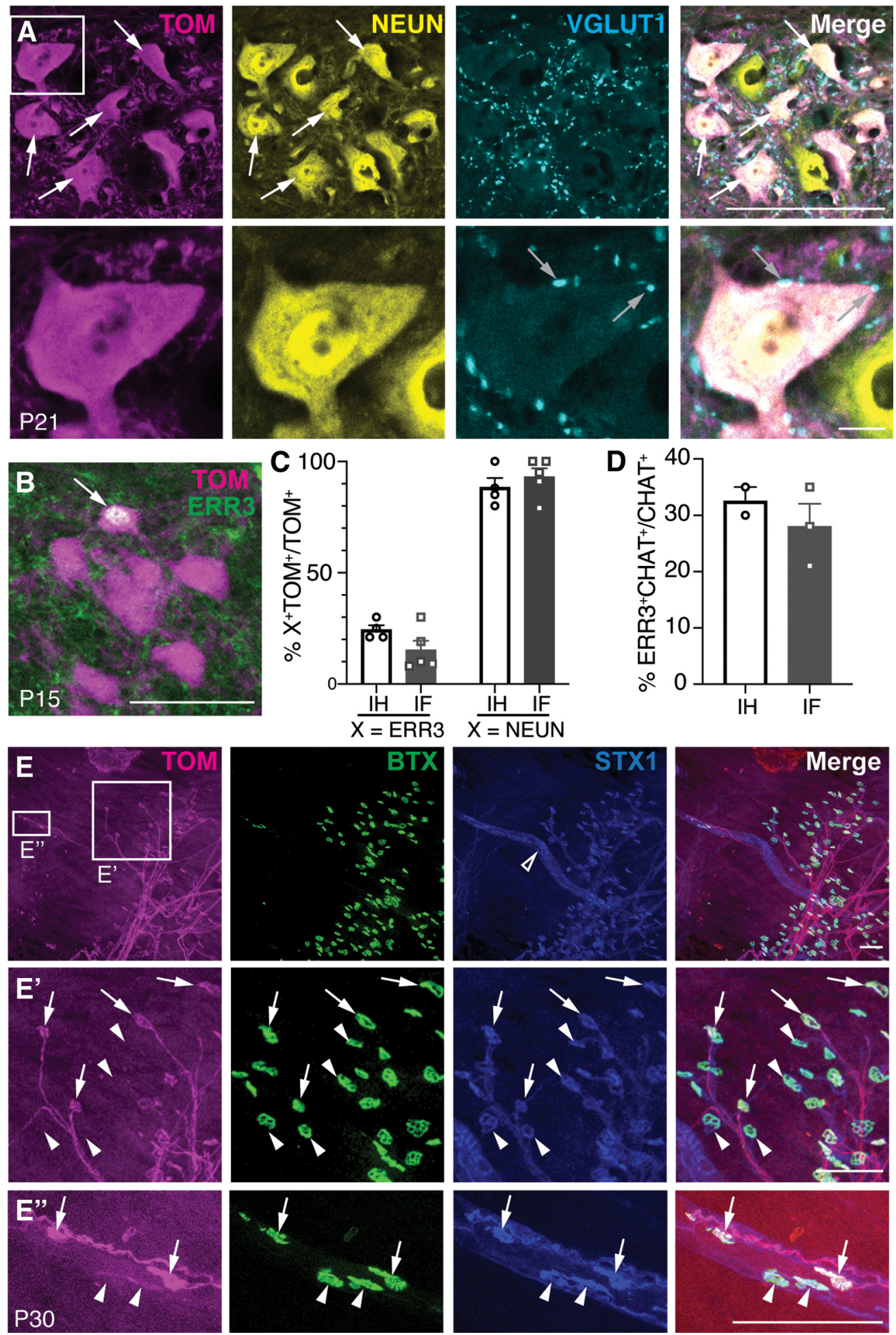

Figure 4. Both $\alpha$-MNs and $\gamma$-MNs are labeled in the Atoh $1^{\text {Cre/+}}$ knock-in mouse. $\boldsymbol{A}, \mathrm{TOM}^{+} \mathrm{MNs}$ in the IF MN pool are NEUN ${ }^{+}$ (arrows) and have closely apposed VGLUT1 ${ }^{+}$boutons (gray arrows). B, Some $\mathrm{TOM}^{+}$IF MNs are also ERR3 ${ }^{+}$(arrow). $\boldsymbol{C}$, Percentage of the $\mathrm{TOM}^{+} \mathrm{MNs}$ in the IH and IF that are ERR3 ${ }^{+}(\gamma-\mathrm{MN}$ marker $)$ or NEUN ${ }^{+}(\alpha-\mathrm{MN}$ marker $)$. D, Percentage of the IH and IF MN pools that are ERR3 ${ }^{+}$. E-E', $\mathrm{TOM}^{+}$axons in the hindpaw lumbrical muscle show the NMJ innervating extrafusal muscle $\left(\boldsymbol{E}^{\prime}\right.$, arrows, $\left.\mathrm{BTX}{ }^{+} \mathrm{STX} 1^{+} \mathrm{TOM}^{+}\right)$. $\mathrm{TOM}^{+}$axons also innervate the intrafusal muscle spindle $(\boldsymbol{E}$, open arrowhead; $\boldsymbol{E}^{\prime \prime}$, arrows, $\left.\mathrm{BTX}^{+} \mathrm{STX} 1^{+} \mathrm{TOM}^{+}\right)$. Arrowheads indicate motor endplates that are $\mathrm{TOM}^{-}$. Scale bars: $100 \mu \mathrm{m} ; 10 \mu \mathrm{m}(\boldsymbol{A}$, inset). 
we were unable to detect any signal at the mRNA level (our unpublished observations). Therefore, to corroborate the labeling of $\mathrm{IH}$ and IF TOM ${ }^{+} \mathrm{MNs}$ in the Atoh $1^{\mathrm{Cre} /+}$ mouse line, we crossed these mice to an Atoh $1^{\text {P2A-FLPo/+ }}$ mouse and FLPo-dependent GFP reporter mouse $\left(R 26^{\text {FSF-EGFP/+ }}\right.$ ) such that neurons from the Atoh $1^{\text {Cre/+ }}$ mouse line were labeled with tdTomato and those from the Atoh $1^{\text {P2A-FLPo/+ }}$ mouse were labeled with EGFP. Strikingly, while the IH and IF MN pools were $\mathrm{TOM}^{+}$, they were clearly GFP $^{-}$(Fig. $1 H$, insets) indicating that the $\mathrm{TOM}^{+} \mathrm{IH}$ and IF MNs are ectopically labeled in the Atoh $1^{\text {Cre/+ }}$ mice, either because of differences in CRE or FLPo recombinase expression themselves or differences in recombination efficiency in the CRE and FLPo lines. Notably, Atoh1-lineage neurons in the intermediate spinal cord had substantial overlap of $\mathrm{GFP}^{+}$and $\mathrm{TOM}^{+}$fluorescence (Fig. $1 \mathrm{H}$, arrowheads) indicating these neurons are reliably from the Atoh 1 -lineage.

\section{Atoh1 ${ }^{\text {Cre/+ }}$ knock-in mice label IH and IF MN pools postnatally}

Because the Atoh $1^{\mathrm{Cre} /+}$ line was labeling IH and IF MNs ectopically, we sought to determine when the ectopic expression occurred during development. We found that at E14.5 when the IH and IF MNs first start expressing the unique markers CPNE4 and FIGN (Mendelsohn et al., 2017), the IH and IF MN pools were not yet $\mathrm{TOM}^{+}$(Fig. $\left.3 C, C^{\prime}\right)$. In contrast, at postnatal time points, we found that $\mathrm{TOM}^{+}$expression started around P3 and gradually increased until it reached $\sim 60 \%$ in the IH and IF MN pools at P22 using the homeobox transcription factor, $\mathrm{HB} 9$ as a marker for MN pools (Arber et al., 1999; P3: IH, $26 \pm 2 \%$, $n=4$, sex was not noted because of young age, four to six half sections $/ n$; IF, $20 \pm 1 \%, n=4$, sex was not noted because of young age, five to six half sections $n$; P7: $\mathrm{IH}, 57 \pm 3 \%, n=3,1: 2 \mathrm{M}: \mathrm{F}$, two to six half sections $/ n$; IF, $40 \pm 2 \%, n=6,2: 4 \mathrm{M}: \mathrm{F}$, three to four half sections $/ n ; \mathrm{P} 15$ : $\mathrm{IH}, 74 \pm 6 \%, n=4,3: 1 \mathrm{M}: \mathrm{F}$, four half sections $/ n$; IF, $71 \pm 3 \%, n=5,3: 2 \mathrm{M}: \mathrm{F}$, four half sections $/ n$; P22: $\mathrm{IH}, 67 \pm 5 \%, n=3,2: 1 \mathrm{M}: \mathrm{F}$, four to eight half sections $/ n$; IF, $62 \pm 2 \%, n=4,1: 3 \mathrm{M}: \mathrm{F}$, four to eight half sections $/ n$; Fig. $3 A-A$ "', $B$ ). In addition, we confirmed that the IF $\mathrm{TOM}^{+}$MNs colocalized with the specific markers CPNE4 and FIGN postnatally (Fig. 3D, $D^{\prime}$ ).

\section{IH and IF MN pools labeled with $A$ toh $1{ }^{\mathrm{Cre} /+}$ knock-in mice are both $\alpha$ and $\gamma$-MNs}

Because Atoh $1^{\text {Cre/+ }} \mathrm{TOM}^{+}$MNs are enriched in only a subset $(\sim 60 \%)$ of IH and IF MNs, we asked whether they were demarcating a specific type of MN ( $\alpha$ or $\gamma$ ). $\alpha$-MNs innervate the striated extrafusal muscle, are marked by the neuronal marker, NEUN, and receive VGLUT1 ${ }^{+}$proprioceptive inputs (Friese et al., 2009; Manuel and Zytnicki, 2011; Ashrafi et al., 2012). $\gamma$-MNs innervate the intrafusal muscle spindles and express estrogen-related receptor $\gamma$ (ERR3 ${ }^{+}$; Friese et al., 2009). Immunostaining for $\alpha$-MN and $\gamma$-MN markers, we found that Atoh 1 Cre/+ $\mathrm{TOM}^{+} \mathrm{MNs}$ were a mixture of both $\alpha-\mathrm{MNs}$ and $\gamma$-MNs (\% ERR3 $^{+}: \mathrm{IH}, 24 \pm 2 \%, n=4,3: 1 \mathrm{M}: \mathrm{F}$, three to four half
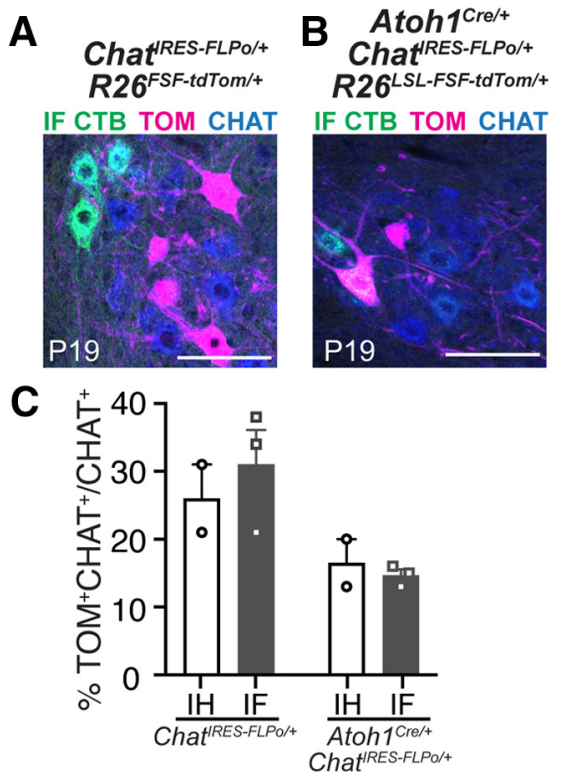

Figure 5. Labeling of IH and IF MNs using an intersectional genetic approach. A, Chat ${ }^{\text {RES-FLPO/+ }}$ mice crossed to a FLPo-dependent tdTomato reporter labels the IF MN pool, identified by injection of CTB into the hindpaw. ChAT Ab identifies the entire MN pool. $\boldsymbol{B}$, The IF MN pool is sparsely labeled using an intersectional cross (Atoh ${ }^{\mathrm{Cre} /+}$; Chat ${ }^{\mathrm{RES}-\mathrm{FLPO} /+}$ ). C, Percentage of the IH and IF MN pools labeled in Chat ${ }^{\prime R E S-F L P O /+}$ mice and Atoh $^{\mathrm{Cre} /+}$; Chat ${ }^{\text {RES-FLPO/+ }}$ mice. IH and IF MN pools were identified by retrograde labeling with CTB. Scale bars: $100 \mu \mathrm{m}$.

sections $/ n$, IF, $15 \pm 4 \%, n=5,3: 2 \mathrm{M}: \mathrm{F}$, two to four half sections $/ n$; \% NEUN ${ }^{+}: \mathrm{IH}, 88 \pm 4 \%, n=4,3: 1 \mathrm{M}: \mathrm{F}$, three half sections $/ n, \mathrm{IF}, 93 \pm 4 \%, n=5,3: 2 \mathrm{M}: \mathrm{F}$, three half sections $/ n$; Fig. $4 A-C$ ). Counts for the percentage of $\mathrm{TOM}^{+}$ neurons that are $\mathrm{ERR}^{+}$or $\mathrm{NEUN}^{+}$were performed on different sets of sections because of the fact that they are both mouse antibodies. Therefore, the percentages do not necessarily add up to $100 \%$ and the error in counts may be attributed to variability between sections. The NEUN ${ }^{+}$ $\mathrm{TOM}^{+}$MNs also received VGLUT1 ${ }^{+}$proprioceptive inputs (Fig. 4A). We confirmed that $\sim 30 \%$ of MNs are $\gamma$-MNs in the IH and IF MN pool as has been reported for other MN pools (IH: $33 \pm 3 \%, n=2,2: 0 \mathrm{M}: \mathrm{F}$, three half sections $/ n$, IF: $28 \pm 4 \%, n=3,2: 1 \mathrm{M}: \mathrm{F}$, two to three half sections $/ n$; Fig. $4 D$; Friese et al., 2009). Therefore, our results suggest that the Atoh $1^{\mathrm{Cre} /+}$ mouse line has a slight preference for labeling $\alpha$-MNs. Furthermore, imaging of the hindpaw lumbrical muscle found $\mathrm{TOM}^{+}$axons innervating both the extrafusal (Fig. 4E', arrows) and intrafusal muscle (Fig. 4E', arrows). $\mathrm{BTX}^{+}$identifies the neuromuscular junctions (NMJs) and syntaxin $\left(\mathrm{STX} 1^{+}\right)$identifies the muscle spindle (Fig. 4E, open arrowhead) and NMJs. Note that not all NMJs are $\mathrm{TOM}^{+}$ (Fig. 4E'-E', arrowheads) consistent with the fact that only $\sim 60 \%$ of the IH and IF MN pools are $\mathrm{TOM}^{+}$.

\section{Intersectional strategy for labeling IH and IF MNs}

Because Atoh1-lineage neurons are glutamatergic and reside in the intermediate spinal cord, we sought a way to isolate specifically the ectopically labeled IH and IF MNs 
using an intersectional strategy crossing Atoh $1^{\mathrm{Cre} /+}$ and Chat ${ }^{I R E S-F L P O /+}$ alleles to the intersectional tdTomato re-

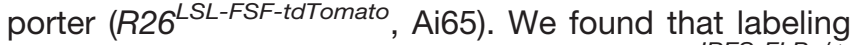
of the IH and IF MNs was sparse in the Chat ${ }^{\text {RESS-FLPO/+ }}$ mouse line alone with only $\sim 30 \%$ of the MN pool being labeled $(\mathrm{IH}, 26 \pm 5 \%, n=2,2: 0 \mathrm{M}: \mathrm{F}$, six half sections $/ n$; IF, $31 \pm 5 \%, n=3,3: 0 \mathrm{M}: \mathrm{F}, 4-10$ half sections $/ n$; Fig. $5 A, C)$. Thus, for the intersection of $A$ toh $^{\mathrm{Cre} /+}$ and Chat ${ }^{\mathrm{RES}-\mathrm{FLPO} /+}$, very few $\mathrm{IH}$ and $\mathrm{IF}$ MNs were labeled $(\mathrm{IH}, 17 \pm 4 \%, n=2,1: 1$ $\mathrm{M}: \mathrm{F}$, two to three sections/n; IF, $15 \pm 1 \%, n=3,2: 1 \mathrm{M}: \mathrm{F}$, three to four half sections $/ n$; Fig. $5 B, C)$. Therefore, this intersectional cross could be useful for sparse labeling of the $\mathrm{IH}$ and IF MN pools.

\section{Discussion}

To understand how dexterous movements of the hand and foot is achieved, we must have some knowledge about the function and circuitry of MNs involved in fine motor control. Thus, obtaining genetic tools in mice that could reproducibly label the $\mathrm{IH}$ and IF MNs could help address questions as to how these neurons differ perhaps in their electrophysiological properties and connectivity compared with MNs innervating limb muscles involved in gross motor control. To this end, we found that MNs that innervate the $\mathrm{IH}$ and IF are labeled in Atoh ${ }^{\mathrm{Cre} /+}$ mice. We found that labeling of the IH and IF MNs is ectopic and occurs postnatally resulting in $\sim 60 \%$ of IH and IF MNs being labeled by approximately three weeks of age with a slight preference for labeling $\alpha$-MNs over $\gamma$-MNs. Here, we discuss some possible uses for the Atoh ${ }^{\mathrm{Cre} /+}$ mouse line for future studies interrogating fine motor control circuits.

If IH and IF MNs could be isolated specifically without labeling other neurons in the nervous system, then a number of genetic tools could be used to either manipulate the activity of these MNs (i.e., optogenetic and/or chemogenetic approaches) or identify the inputs to these MNs (i.e., transsynaptic rabies virus tracing). One approach to isolate $\mathrm{IH}$ and IF MNs would be to use an intersectional genetic strategy crossing the Atoh $1^{\mathrm{Cre} /+}$ mice to mice expressing FLPo recombinase in the IH and IF MNs, but not in other overlapping Atoh $1^{\text {Cre/+ }}$ domains. We attempted an initial intersectional cross of Atoh $1^{\text {Cre/+ }}$ to Chat ${ }^{I R E S-F L P O /+}$ and found sparse labeling of the IH and IF MNs because of inefficient recombination in these MNs by the Chat ${ }^{\text {RES }-F L P O /+}$ line. While this particular intersectional cross could be useful for sparse labeling of the IH and IF MNs for anatomic studies, one must keep in mind that Atoh $1^{\mathrm{Cre} /+}$ and Chat ${ }^{\mathrm{RES}-\mathrm{FLPO} /+}$ intersect in other areas of the central nervous system such as occasionally in other MN pools (Fig. 2) and in Atoh1-lineage cholinergic neurons in the pedunculopontine tegmentum (PPTg) and the lateral dorsal tegmentum (LDTg) (our unpublished observations; Rose et al., 2009). Thus, an alternate cross is required to separate out the IH and IF MNs.

Moving forward, we propose that other FLPo-recombinase lines could be used in conjunction with the Atoh $1^{\mathrm{Cre} /+}$ mouse line to isolate the IH and IF MNs. For example, FLPo driven by $\mathrm{Hb9}$, Fign, or Cpne4, which we have shown colocalize with the $\mathrm{IH}$ and IF MNs labeled in the Atoh $1^{\mathrm{Cre} /+}$ line, but not in other Atoh1-lineage neurons in the intermediate spinal cord (Fig. 3), would be promising candidates. $\mathrm{Hb9}$, however, has the same caveat as Chat in that other MN pools throughout the spinal cord may intersect with Atoh $1^{\mathrm{Cre} /+}$ expression. IH and IF MNs were found to contain a repertoire of unique molecular markers compared with neighboring limb-innervating MNs suggesting a unique developmental program (Mendelsohn et al., 2017). Indeed, the transcriptional landscape in IH and IF MNs appears to preferentially activate the CRE recombinase in Atoh $1^{\mathrm{Cre} /+}$ mice. Of these unique molecular markers, other candidate genes include Ecrg4, Reg3b, Serpinf1, and Pirt (Mendelsohn et al., 2017), although these would need further characterization of spatial and temporal overlap with the Atoh $1^{\mathrm{Cre} /+}$ line to determine their suitability for future studies. Furthermore, Osmr and Col8a1 could be considered for exploration of specifically IF MNs (Mendelsohn et al., 2017).

An alternate strategy would be to use viruses to restrict labeling to just the IH or IF MN pools. For example, the Atoh $1^{\text {Cre/+ }}$ line could be crossed to an intersectional line expressing a reporter of choice (i.e., fluorescent, optogenetic, or chemogenetic reporter) and an AAV-FLPo injected into either the spinal cord or in the forepaw or hindpaw to be taken up retrogradely to the IH and IF MNs. However, a caveat of this approach is the potential damage to the spinal cord or muscles of the forepaw and hindpaw because of the needle injection. Thus, appropriate injection controls would be needed with this approach.

Altogether, we present here that the Atoh $1^{\mathrm{Cre} /+}$ mouse consistently labels $\mathrm{MNs}$ of the $\mathrm{IH}$ and IF and that the Atoh $1^{\mathrm{Cre} /+}$ mouse could be used to probe the function and connectivity of MNs in fine motor control. Our findings also serve as a cautionary tale of relying on CRErecombinase mouse lines to faithfully report endogenous gene expression and speak to the need for careful follow-up experiments to appropriately interpret reporter expression.

\section{References}

Allaway KC, Muñoz W, Tremblay R, Sherer M, Herron J, Rudy B, Machold R, Fishell G (2020) Cellular birthdate predicts laminar and regional cholinergic projection topography in the forebrain. Elife 9: e63249.

Arber S, Han B, Mendelsohn M, Smith M, Jessell TM, Sockanathan S (1999) Requirement for the homeobox gene Hb9 in the consolidation of motor neuron identity. Neuron 23:659-674.

Ashrafi $S$, Lalancette-Hébert $M$, Friese A, Sigrist $M$, Arber $S$, Shneider NA, Kaltschmidt JA (2012) Wnt7A identifies embryonic $\gamma$-motor neurons and reveals early postnatal dependence of $\gamma$-motor neurons on a muscle spindle-derived signal. J Neurosci 32:8725-8731.

Bermingham NA, Hassan BA, Wang VY, Fernandez M, Banfi S, Bellen HJ, Fritzsch B, Zoghbi HY (2001) Proprioceptor pathway development is dependent on Math1. Neuron 30:411-422.

Dempsey-Jones H, Wesselink DB, Friedman J, Makin TR (2019) Organized toe maps in extreme foot users. Cell Rep 28:27482756.e4.

Friese A, Kaltschmidt JA, Ladle DR, Sigrist M, Jessell TM, Arber S (2009) Gamma and alpha motor neurons distinguished by expression of transcription factor Err3. Proc Natl Acad Sci USA 106:13588-13593.

Gowan K, Helms AW, Hunsaker TL, Collisson T, Ebert PJ, Odom R, Johnson JE (2001) Crossinhibitory activities of Ngn1 and Math1 allow specification of distinct dorsal interneurons. Neuron 31:219232. 
Jacobi AM, Rettig GR, Turk R, Collingwood MA, Zeiner SA, Quadros RM, Harms DW, Bonthuis PJ, Gregg C, Ohtsuka M, Gurumurthy CB, Behlke MA (2017) Simplified CRISPR tools for efficient genome editing and streamlined protocols for their delivery into mammalian cells and mouse zygotes. Methods 121-122:16-28.

Kaplan A, Spiller KJ, Towne C, Kanning KC, Choe GT, Geber A, Akay T, Aebischer P, Henderson CE (2014) Neuronal matrix metalloproteinase- 9 is a determinant of selective neurodegeneration. Neuron 81:333-348

Kim JH, Lee SR, Li LH, Park HJ, Park JH, Lee KY, Kim MK, Shin BA, Choi SY (2011) High cleavage efficiency of a 2A peptide derived from porcine teschovirus- 1 in human cell lines, zebrafish and mice. PLoS One 6:e18556.

Lai HC, Seal RP, Johnson JE (2016) Making sense out of spinal cord somatosensory development. Development 143:3434-3448.

Lu QR, Sun T, Zhu Z, Ma N, Garcia M, Stiles CD, Rowitch DH (2002) Common developmental requirement for Olig function indicates a motor neuron/oligodendrocyte connection. Cell 109:75-86.

Madisen L, Zwingman TA, Sunkin SM, On SW, Zariwala HA, Gu H, $\mathrm{Ng}$ LL, Palmiter RD, Hawrylycz MJ, Jones AR, Lein ES, Zeng $\mathrm{H}$ (2010) A robust and high-throughput Cre reporting and characterization system for the whole mouse brain. Nat Neurosci 13:133140.

Madisen L, Garner AR, Shimaoka D, Chuong AS, Klapoetke NC, Li L, van der Bourg A, Niino Y, Egolf L, Monetti C, Gu H, Mills M, Cheng A, Tasic B, Nguyen TN, Sunkin SM, Benucci A, Nagy A, Miyawaki A, Helmchen F, et al. (2015) Transgenic mice for intersectional targeting of neural sensors and effectors with high specificity and performance. Neuron 85:942-958.

Manuel M, Zytnicki D (2011) Alpha, beta and gamma motoneurons: functional diversity in the motor system's final pathway. J Integr Neurosci 10:243-276.

Mendelsohn AI, Dasen JS, Jessell TM (2017) Divergent hox coding and evasion of retinoid signaling specifies motor neurons innervating digit muscles. Neuron 93:792-805.e4.

Miura H, Quadros RM, Gurumurthy CB, Ohtsuka M (2018) EasiCRISPR for creating knock-in and conditional knockout mouse models using long ssDNA donors. Nat Protoc 13:195-215.

Miyoshi G, Hjerling-Leffler J, Karayannis T, Sousa VH, Butt SJ, Battiste J, Johnson JE, Machold RP, Fishell G (2010) Genetic fate mapping reveals that the caudal ganglionic eminence produces a large and diverse population of superficial cortical interneurons. $J$ Neurosci 30:1582-1594.

Pop IV, Espinosa F, Goyal M, Mona B, Landy MA, Ogujiofor OW, Dean KM, Gurumurthy CB, Lai HC (2020) Evidence for genetically distinct direct and indirect spinocerebellar pathways mediating proprioception. bioRxiv. doi: https://doi.org/10.1101/2020.08.17 .254607 .
Quadros RM, Miura H, Harms DW, Akatsuka H, Sato T, Aida T, Redder R, Richardson GP, Inagaki Y, Sakai D, Buckley SM, Seshacharyulu P, Batra SK, Behlke MA, Zeiner SA, Jacobi AM, Izu Y, Thoreson WB, Urness LD, Mansour SL, et al. (2017) EasiCRISPR: a robust method for one-step generation of mice carrying conditional and insertion alleles using long ssDNA donors and CRISPR ribonucleoproteins. Genome Biol 18:92.

Rose MF, Ahmad KA, Thaller C, Zoghbi HY (2009) Excitatory neurons of the proprioceptive, interoceptive, and arousal hindbrain networks share a developmental requirement for Math1. Proc Natl Acad Sci USA 106:22462-22467.

Sakai K, Miyazaki J (1997) A transgenic mouse line that retains Cre recombinase activity in mature oocytes irrespective of the cre transgene transmission. Biochem Biophys Res Commun 237:318324.

Sakai N, Insolera R, Sillitoe RV, Shi SH, Kaprielian Z (2012) Axon sorting within the spinal cord marginal zone via Robo-mediated inhibition of $\mathrm{N}$-cadherin controls spinocerebellar tract formation. $\mathrm{J}$ Neurosci 32:15377-15387.

Schindelin J, Arganda-Carreras I, Frise E, Kaynig V, Longair M, Pietzsch T, Preibisch S, Rueden C, Saalfeld S, Schmid B, Tinevez JY, White DJ, Hartenstein V, Eliceiri K, Tomancak P, Cardona A (2012) Fiji: an open-source platform for biological-image analysis. Nat Methods 9:676-682.

Shubin N, Tabin C, Carroll S (1997) Fossils, genes and the evolution of animal limbs. Nature 388:639-648.

Sustaita D, Pouydebat E, Manzano A, Abdala V, Hertel F, Herrel A (2013) Getting a grip on tetrapod grasping: form, function, and evolution. Biol Rev Camb Philos Soc 88:380-405.

Tennant KA, Asay AL, Allred RP, Ozburn AR, Kleim JA, Jones TA (2010) The vermicelli and capellini handling tests: simple quantitative measures of dexterous forepaw function in rats and mice. $J$ Vis Exp. Advance online publication. Retrieved July 21, 2010. doi: 10.3791/2076.

Watson C, Paxinos G, Kayalioglu G (2009) The spinal cord: a Christopher and Dana Reeve Foundation text and atlas. New York: Academic Press.

Yang H, Xie X, Deng M, Chen X, Gan L (2010) Generation and characterization of Atoh1-Cre knock-in mouse line. Genesis 48:407413.

Yoshida Y, Isa T (2018) Neural and genetic basis of dexterous hand movements. Curr Opin Neurobiol 52:25-32.

Yuengert R, Hori K, Kibodeaux EE, McClellan JX, Morales JE, Huang TW, Neul JL, Lai HC (2015) Origin of a non-Clarke's column division of the dorsal spinocerebellar tract and the role of caudal proprioceptive neurons in motor function. Cell Rep 13:1258-1271. 